\title{
DISCRETE-TIME SLIDING MODE CONTROL FOR A CLASS OF UNDERACTUATED MECHANICAL SYSTEMS WITH BOUNDED DISTURBANCES
}

\author{
NGUYEN DINH THAT \\ Faculty of Marine Electrical and Electronics Engineering, Vietnam Maritime University \\ Faculty of Engineering and Information Technology, University of Technology, Sydney, \\ NSW 2007; thatkd@vimaru.vn
}

Tóm tắt. Bài báo đề xuất một phương pháp thiết kế bộ điều khiển tựa trượt gián đoạn cho một lớp hệ hụt cơ cấu chấp hành có xét đến ảnh hưởng của nhiễu bị chặn. Dựa trên phương pháp hàm Lyapunov, một điều kiện đủ cho sự tồn tại một mặt trượt ổn định được đưa ra dưới dạng bất đẳng thức ma trận tuyến tính. Điều kiện này cũng đảm bảo rằng ảnh hưởng của nhiễu bị chặn sẽ bị loại bỏ khi hệ ở trong chế độ trượt. Mặt khác, khi trong chế độ trượt, quỹ đạo trạng thái của hệ hội tụ mũ tới một hình cầu bán kính nhỏ nhất. Bộ điều khiển tựa trượt gián đoạn sau đó được đề xuất để đưa quỹ đạo trạng thái của hệ vào mặt trượt trong thời gian hữu hạn và duy trì quỹ đạo trên mặt trượt. Một nghiên cứu trên robot Pendubot được đưa ra để kiểm chứng tính khả thi của bộ điều khiển được đề xuất.

Từ khóa. Hệ hụt cơ cấu chấp hành, điều khiển trượt, pendubot, nhiễu bị chặn, Lyapunov, bất đẳng thức ma trận tuyến tính.

\begin{abstract}
This article addresses the problem of discrete-time quasi-sliding mode control for a class of underactuated mechanical systems in the presence of bounded external disturbances. Based on the Lyapunov functional method, a sufficient condition for the existence of a stable sliding surface is derived in terms of a linear matrix inequality (LMI). This condition also guarantees that the effects of external disturbances can be suppressed when the system is considered in the sliding mode. Moreover, in the induced sliding dynamics, all the state trajectories are exponentially convergent to a ball whose radius can be minimized. A robust discrete-time quasi-sliding mode controller is then proposed to drive system state trajectories towards the sliding surface infinite time and maintain it thereafter subsequent time. A case study of the Pendubot is provided to illustrate the feasibility of the proposed approach.
\end{abstract}

Key words. Underactuated mechanical system, sliding mode control, pendubot, bounded disturbance, Lyapunov, linear matrix inequality (LMI).

\section{INTRODUCTION}

Recently, there have been increasingly efforts devoted to the control problem of underactuated mechanical systems which have fewer actuators than the degrees of freedom (see, e.g, [1] and references therein). In general, this can be a natural design due to mechanical 
constraints or an intentional purpose for reducing the cost. Due to undesired properties of their dynamics including nonlinearities, non-holonomic constraints and couplings, the control of underactuated mechanical systems is more difficult than fully actuated ones. Based on feedback linearization methods in which the dynamic system is transformed into a strict feedback form, a linear quadratic regulator (LQR) was proposed to balance the Acrobot [2]. A hybrid controller was developed for the swing-up and balance control problem of the Pendubot [3, 4]. By using the energy-based methods, some approaches were proposed for set-point regulation of underactuated mechanical systems. The main idea of energy-based control methods is to regulate the total energy of system to the equivalent value of a desired equilibrium state. An energy-based control strategy was introduced to the problem of swing-up and stabilization of the Pendubot [5, 6]. In [7, a new formulation of passivity-based control was developed for stabilization of a class of underactuated mechanical systems via interconnection and damping assignment. Recently, some improved results was reported in [8, 9, 10]. However, the main drawback of the energy-based methods is that it requires the number of the relative degrees of the system to be less than two. To overcome this, a backstepping technique was proposed to transform the system into a new recursive form which the energy-based methods can be easily applied (see [11, 12, 13]). The controlled Lagrangian method is another approach for the control of underactuated mechanical systems. The merit of this approach is to modify the generalised inertia matrix and potential energy functions of the uncontrolled dynamics to controlled Lagrangian [14, 15].

However, there remaining interesting questions as how to deal with the parameter variations and external disturbances, which are unavoidable in the control of underactuated mechanical systems. In this context, sliding mode control (SMC) has been considered as a promising method, see, e.g., [16] and the references therein. The main purpose of the SMC is first to design a stable sliding surface with desired performance characteristics. Then, the discontinuous control is designed to drive the state trajectory towards the sliding surface and maintain it on this surface over time. The dynamic characteristics of the resulting closed-loop control system will be mainly determined by the sliding surface design. A sliding mode control for trajectory tracking control of autonomous surface vessels was presented in [17] by using a first-order surface and a second-order surface in terms of the surge tracking errors and the sway tracking errors, respectively. In [18], a robust sliding mode controller was proposed for velocity tracking of the mobile-wheeled inverted-pendulum that is subject to parameter uncertainties and external disturbances. The asymptotically stable of the closed-loop systems were achieved through the selection of sliding-surface parameters. Similar improvements were reported in [20, 21, 24]. Along with advantages including cost-effectiveness and high flexibility of digital computers, there have been considerable attention to the problem of discrete-time controller design in embedded control applications. However, it appears that the problem of robust discrete-time quasi-sliding mode control for underactuated mechanical systems with external disturbances has not received much attention.

Motivated by That (2013), who investigated the problem of discrete-time sliding mode control for the Pendubot in the presence of bounded disturbances, we further consider the problem of discrete-time quasi-sliding mode control design for a class of underactuated mechanical systems with bounded external disturbances. The main contribution of this study are threefold as follows:

- To further analyze the effects of the unmodeled dynamics and external disturbances and model them as bounded disturbances in discrete-time model of system dynamics.

- To derive a sufficient condition for the existence of a stable sliding surface in terms of 
a linear matrix inequality and guarantee that the reduced-order sliding mode dynamics are bounded within a ball whose radius can be minimized.

- To propose a discrete-time quasi-sliding mode controller to drive the system trajectories towards the sliding surface in finite time and maintain it on the surface afterwards.

The paper is organized as follows. Section 2 presents the dynamics of underactuatated mechanical systems, the problem statement and some preliminaries. The main results are included in Section 3, in which the sliding surface is first designed. A discrete-time quasi-sliding mode control law is then proposed to guarantee that the system state trajectories will reach the sliding surface in finite time and maintain it there afterwards. A case study of the Pendubot is provided in Section IV. Finally, Section 5 concludes the paper.

Notations: In this paper, $\mathbb{R}^{n}$ denotes the $n$-dimensional space and $\|$.$\| is its vector norm; \mathbb{R}^{n \times m}$ denotes the space of all matrices of $(n \times m)$-dimension; $I$ and 0 represent identity matrix and zero matrix, respectively, with appropriate dimensions. $A^{T}$ denotes the transpose of matrix $A ; \lambda(A)$ denotes the set of all eigenvalues of matrix $A ; \lambda_{\min }(A)=\min \{\operatorname{Re} \lambda \mid \lambda \in \lambda(A)\} ;$ and $(*)$ in a matrix implies the symmetric term. Throughout the paper, inequalities between real vectors are understood componentwise and matrix operator o denotes the Hadamard product, i.e., $(A \circ B)_{i, j}=A_{i, j} . B_{i, j}$.

\section{PROBLEM STATEMENT AND PRELIMINARIES}

Consider the generic motion equation for a class of underactuated mechanical systems in the form

$$
M(q) \ddot{q}+C(q, \dot{q}) \dot{q}+g(q)+v(t)=F,
$$

where $M(q)=M(q)^{T} \in \mathbb{R}^{n \times n}$ is the symmetric inertial matrix, $C(q, \dot{q}) \in \mathbb{R}^{n \times n}$ and $g(q) \in \mathbb{R}^{n}$ are, respectively, the matrices containing the centrifugal-Coriolis and the gravitational terms; $v(t)$ is the vector of the effects of unmodeled dynamics and external disturbances; $F \in \mathbb{R}^{n}$ is the vector of the input control forces applied to the links and $q \in \mathbb{R}^{n}$ is the vector of generalized coordinates.

It should be noted that when the number of non-zero control inputs in vector $F$ is less than the degrees of freedom to be controlled, then system (1) becomes an underactuated mechanical system. Assume that the number of actuators for an $n$ degree-of-freedom of underactuated mechanical system (1) is $m$, where $m<n$. The vector of generalised coordinates can be partitioned as $q^{T}=\left[\begin{array}{ll}q_{a}^{T} & q_{u}^{T}\end{array}\right]$, where $q_{a} \in \mathbb{R}^{m}$ is the vector of actuated generalized coordinates and $q_{u} \in \mathbb{R}^{n-m}$ is the vector of unactuated generalized coordinates. The vector of control forces $F$ is also written as $F^{T}=\left[\begin{array}{ll}u^{T} & 0\end{array}\right]$ where $u(t) \in \mathbb{R}^{m}$. Then, system (1) can be rewritten in the form

$$
\left[\begin{array}{ll}
M_{a a}\left(q_{u}\right) & M_{a u}\left(q_{u}\right) \\
M_{u a}\left(q_{u}\right) & M_{u u}\left(q_{u}\right)
\end{array}\right]\left[\begin{array}{c}
\ddot{q}_{a} \\
\ddot{q}_{u}
\end{array}\right]+\left[\begin{array}{c}
C_{a u}\left(q_{u}, \dot{q}_{u}\right) \\
C_{u u}\left(q_{u}, \dot{q}_{u}\right)
\end{array}\right]\left[\begin{array}{c}
\dot{q}_{a} \\
\dot{q}_{u}
\end{array}\right]+\left[\begin{array}{c}
g_{a}\left(q_{a}\right) \\
g_{u}\left(q_{u}\right)
\end{array}\right]+\left[\begin{array}{c}
v_{a}(t) \\
v_{u}(t)
\end{array}\right]=\left[\begin{array}{c}
u \\
0
\end{array}\right]
$$

Since $M(q)$ is a symmetric matrix, we therefore obtain $\ddot{q}$ from (1) as follows

$$
\ddot{q}=\left[\begin{array}{c}
\ddot{q}_{a} \\
\ddot{q}_{u}
\end{array}\right]=M(q)^{-1}(F-C(q, \dot{q}) \dot{q}-g(q)-v(t)) .
$$


Let us define the following state variables

$$
x=\left[\begin{array}{l}
x_{1} \\
x_{2}
\end{array}\right]=\left[\begin{array}{c}
q \\
\dot{q}
\end{array}\right],
$$

then, system (1) can thus be rewritten as

$$
\dot{x}=f(x)+g_{1}(x) u+g_{2}(x) v(t),
$$

where

$$
\begin{gathered}
f(x)=\left[\begin{array}{c}
x_{2} \\
-M^{-1}\left(x_{1}\right)\left(C\left(x_{1}, x_{2}\right) x_{2}+g\left(x_{1}\right)\right)
\end{array}\right], \\
g_{1}(x)=\left[\begin{array}{c}
0 \\
-M^{-1}\left(x_{1}\right)
\end{array}\right], g_{2}(x)=\left[\begin{array}{c}
0 \\
M^{-1}\left(x_{1}\right)
\end{array}\right] .
\end{gathered}
$$

Once a nominal solution (i.e., $x_{0}$ ) to the nonlinear equation (5) has been obtained, the linearized perturbation equation of underactuated mechanical system (1) is

$$
\dot{\bar{x}}(t)=A \bar{x}(t)+B u(t)+D v(t),
$$

where the Jacobian matrices are defined as follows

$$
A=\left.\frac{\partial f}{\partial x}\right|_{x=x_{0}}, B=\left.g_{1}\right|_{x=x_{0}}, D=\left.g_{2}\right|_{x=x_{0}} .
$$

To obtain a discrete-time state space model from a continuous-time state space model, the following discretization approximation is adopted

$$
\dot{\bar{x}}_{i} \approx \frac{\bar{x}_{i}(k+1)-\bar{x}_{i}(k)}{T_{s}},
$$

where $T_{s}$ is the sampling period. The discrete-time model of the system is therefore obtained as

$$
\bar{x}(k+1)=\bar{A} \bar{x}(k)+\bar{B} u(k)+\bar{D} v(k),
$$

where $\bar{A}=I+T_{s} A, \bar{B}=T_{s} B, \bar{D}=T_{s} D$. Without loss of generality, the vector of the effects of unmodeled dynamics and external disturbances $v(t)$ is assumed to be bounded as

$$
v^{T}(k) v(k) \leq \bar{v}_{p}^{2}
$$

where $\bar{v}_{p}$ is a known positive scalar. To facilitate the development of our approach, a state transformation $z(k)=T \bar{x}(k)=\left[\begin{array}{c}z_{1}(k) \\ z_{2}(k)\end{array}\right]$ is introduced, such that $T B=\left[\begin{array}{c}0 \\ B_{2}\end{array}\right]$, where $B_{2}$ is a non-singular matrix. System (8) can therefore be transformed into the following regular form:

$$
z(k+1)=\bar{A} z(k)+\bar{B} u(k)+\bar{D} v(k),
$$

where

$$
\bar{A}=T A T^{-1}=\left[\begin{array}{ll}
A_{11} & A_{12} \\
A_{21} & A_{22}
\end{array}\right], \bar{D}=T D=\left[\begin{array}{c}
D_{1} \\
D_{2}
\end{array}\right], \bar{B}=T B .
$$

System (10) can also be rewritten in the form of

$$
\begin{aligned}
& z_{1}(k+1)=A_{11} z_{1}(k)+A_{12} z_{2}(k)+D_{1} v(k), \\
& z_{2}(k+1)=A_{21} z_{1}(k)+A_{22} z_{2}(k)+B_{2} u(k)+D_{2} v(k) .
\end{aligned}
$$


This transformation is highly desirable as state equation $z_{1}(k+1)$ will become independent of the control input. Our purpose is first to design a stable sliding surface for system (10) such that the desired performance can be achieved while the system states still remain on the sliding surface. A sufficient condition is derived in terms of a linear matrix inequality to guarantee that the effects of unmodeled dynamics and external disturbances can be suppressed when the system is in the sliding mode and the reduced-order sliding mode dynamics are exponentially convergent to a ball whose radius can be minimized. Finally, a discrete-time quasi-sliding mode controller is proposed to drive the system states towards the sliding surface.

\section{MAIN RESULTS}

\subsection{Switching surface design and existence problem}

In this subsection, the sliding surface design with desired performance characteristics and the existence problem are considered. A sufficient condition is derived to guarantee that the effects of external disturbances can be suppressed and reduced-order sliding-mode dynamics exponentially converge within a ball in the sliding mode. The sliding function is proposed as follows

$$
s(k)=\bar{C} z(k)=\left[\begin{array}{ll}
-C & I
\end{array}\right] z(k)=-C z_{1}(k)+z_{2}(k),
$$

where $\bar{C}=\left[\begin{array}{ll}-C & I\end{array}\right]$ in which $C \in \mathbb{R}^{m \times(n-m)}$ is a constant matrix to be designed.

During the sliding motion, we have $s(k)=0$ so that $z_{2}(k)=C z_{1}(k)$. Reduced-order sliding dynamics system can thus be obtained as

$$
z_{1}(k+1)=\left[A_{11}+A_{12} C\right] z_{1}(k)+D_{1} v(k) .
$$

Before presenting our main results, the following definition and lemmas are introduced.

For any positive scalar $r>0$, let the ball $\mathcal{B}(0, r)$ be defined by

$$
\mathcal{B}(0, r)=\left\{z_{1} \in \mathbb{R}^{n-m}:\left\|z_{1}\right\| \leq r\right\} .
$$

Definition 1. For a given positive scalar $\delta>0$, the solution of system $(13)$ is exponentially convergent to a ball $B(0, r)=\left\{z_{1} \in \mathbb{R}^{n-m}:\left\|z_{1}\right\| \leq r\right\}$ with rate $\gamma>0$ if there exists a non-negative functional $\eta(\delta)>0$ such that the following inequality holds

$$
\left\|z_{1}(k)\right\| \leq r+\eta(\delta) e^{-\gamma k}, \text { for all } k \geq 0 .
$$

In case of without disturbance (i.e. $v(k)=0$, for all $k \geq 0$,) the solution of system 13 is said to be $\gamma$-exponentially stable.

Lemma 1. (Gu, 2000) Given constant matrices $X, Y, Z$ with appropriate dimensions satisfying $X=X^{T}, Y=Y^{T}>0$. Then $X+Z^{T} Y^{-1} Z<0$ if and only if

$$
\left[\begin{array}{cc}
X & Z^{T} \\
Z & -Y
\end{array}\right]<0 \text { or }\left[\begin{array}{cc}
-Y & Z \\
Z^{T} & X
\end{array}\right]<0
$$

Lemma 2. If there exist a scalar $\alpha>1$ and a positive definite function $V(k)$ satisfy

$$
\Delta V(k+1)+\left(1-\alpha^{-1}\right) V(k)-\left(1-\alpha^{-1}\right) v^{T}(k) v(k) \leq 0,
$$


then the following inequality holds

$$
V(k) \leq \bar{v}_{p}^{2}+V(0) e^{-\gamma k}, \forall k \geq 0,
$$

where $\gamma=\ln (\alpha)>0$. Therefore, we obtain the following inequality

$$
\lim _{k \rightarrow \infty} \sup V(k) \leq \bar{v}_{p}^{2}
$$

Proof. From (9) and (16), we have

$$
\begin{aligned}
V(k+1) \leq & \alpha^{-1} V(k)+\left(1-\alpha^{-1}\right) \bar{v}_{p}^{2} \\
\leq & \alpha^{-2} V(k-1)+\left(1-\alpha^{-2}\right) \bar{v}_{p}^{2} \\
& \cdots \\
\leq & \alpha^{-(k+1)} V(0)+\left(1-\alpha^{-(k+1)}\right) \bar{v}_{p}^{2} .
\end{aligned}
$$

Since $\alpha>1$, thus

$$
V(k) \leq \bar{v}_{p}^{2}+V(0) e^{-\gamma k}
$$

Consequently, we have

$$
\lim _{k \rightarrow \infty} \sup V(k) \leq \bar{v}_{p}^{2}
$$

This completes the proof.

Theorem 1. For given positive scalars $\bar{v}_{p}$ and $\alpha>1$, if there exist a matrix $K$ and a symmetric positive-definite matrix $X$, such that the following linear matrix inequality is satisfied

$$
\left[\begin{array}{ccc}
-\alpha^{-1} X & \star & \star \\
0 & -\left(1-\alpha^{-1}\right) I_{p} & \star \\
A_{11} X+A_{12} K & D_{1} & -X
\end{array}\right]<0,
$$

then the state trajectories of reduced-order system (13) are exponentially convergent within a ball $B(0, r)=\left\{z_{1} \in \mathbb{R}^{n-m}|:| \mid z_{1} \| \leq r\right\}$, where the radius $r$ is computed as

$$
r=\frac{\bar{v}_{p}}{\sqrt{\lambda_{\min }\left(X^{-1}\right)}} .
$$

Moreover, the design switching gain $C$ in (12) is obtained explicitly as

$$
C=K X^{-1}
$$

Proof. Let $X>0$ be a solution of 19 , denote $P=X^{-1}$. We consider the following Lyapunov function

$$
V=z_{1}^{T}(k) P z_{1}(k) .
$$

Then, by taking the difference of functional 22 along the solution of system 13 , we obtain

$$
\begin{aligned}
\Delta V(k) & =z_{1}^{T}(k+1) P z_{1}(k+1)-z_{1}^{T}(k) P z_{1}(k) \\
& =\left[\left(A_{11}+A_{12} C\right) z_{1}(k)+D_{1} v(k)\right]^{T} P\left[\left(A_{11}+A_{12} C\right) z_{1}(k)+D_{1} v(k)\right] \\
& -\alpha^{-1} z_{1}(k) P z_{1}(k)-\left(1-\alpha^{-1}\right) V(k) .
\end{aligned}
$$


From above, we obtain

$$
\Delta V(k)+\left(1-\alpha^{-1}\right) V(k)-\left(1-\alpha^{-1}\right) v^{T}(k) v(k) \leq \zeta^{T}(k)\left[\Phi+\Psi P \Psi^{T}\right] \zeta(k),
$$

where $\zeta^{T}(k)=\left[\begin{array}{ll}z_{1}^{T}(k) & v^{T}(k)\end{array}\right]$ and

$$
\Phi=\left[\begin{array}{cc}
-\alpha^{-1} P & \star \\
0 & -\left(1-\alpha^{-1}\right) I_{p}
\end{array}\right], \Psi=\left[\begin{array}{c}
\left(A_{11}+A_{12} C\right)^{T} \\
D_{1}^{T}
\end{array}\right] .
$$

Thus

$$
\Delta V(k)+\left(1-\alpha^{-1}\right) V(k)-\left(1-\alpha^{-1}\right) v^{T}(k) v(k) \leq 0,
$$

if the following inequality holds

$$
\Phi+\Psi P \Psi^{T}<0
$$

Now by using the Schur inequality in Lemma 1, 24 can be brought to the form,

$$
\left[\begin{array}{ccc}
-\alpha^{-1} P & \star & \star \\
0 & -\left(1-\alpha^{-1}\right) I_{p} & \star \\
\left(A_{11}+A_{12} C\right) & D_{1} & -P^{-1}
\end{array}\right]<0 .
$$

By multiplying equation 25 from the right and the left by $\operatorname{diag}\left(P^{-1}, I, I\right)$ and its transpose, respectively, we obtain

$$
\left[\begin{array}{ccc}
-\alpha^{-1} P^{-1} & \star & \star \\
0 & -\left(1-\alpha^{-1}\right) I_{p} & \star \\
\left(A_{11}+A_{12} C\right) P^{-1} & D_{1} & -P^{-1}
\end{array}\right]<0,
$$

By defining $C=K P=K X^{-1}$, one can obtain inequality (19). From Lemma 2, we have $\lim _{k \rightarrow \infty} \sup V(k) \leq \bar{v}_{p}^{2}$, which yields $z_{1}^{T}(k) P z_{1}(k) \leq \bar{v}_{p}^{2}$. By using the spectral properties of symmetric positive-definite matrix, we have

$$
\lambda_{\min }(P)\left\|z_{1}(k)\right\|^{2} \leq \bar{v}_{p}^{2}
$$

This means that $\left\|z_{1}(k)\right\| \leq r$. Thus, the sliding dynamics in the sliding mode are bounded within a ball with radius $r$. The proof is completed.

Remark 1. It is worth mentioning that in this paper, the sliding function in 12 is not required to satisfy any condition, i.e. to directly connected to the system output, $s(k)=$ $F y(k), y(k)=C x(k)$, where the selection of the sliding function $s(k)$ may cause a difficulty in the determination of a matrix $F$. Here, the switching gain is directly obtained from the solution of linear matrix inequality (19). This makes our proposed approach more applicable than that in literature.

Remark 2. It should be noted that the matrix inequality 19 obtained in Theorem 1 contains only one non-convex scalar $\alpha$. However, for a given scalar $\alpha$, it becomes a linear matrix inequality (LMI) and can be solved efficiently in by using a one-dimensional search method, combined with Matlab's LMI Toolbox.

Remark 3. It is easy to see that the radius of the ball $\mathcal{B}(0, r)=\left\{z_{1} \in \mathbb{R}^{n}:\left\|z_{1}\right\| \leq r\right\}$ is determined by $r=\frac{\bar{v}_{p}}{\sqrt{\delta}}$, where $\delta=\lambda_{\min }\left(X^{-1}\right)$. Thus, to find the smallest possible radius $r$, 
one may propose a simple optimization problem stated in That et al. 2013 to maximize $\delta$ subject to $\delta I \leq X^{-1}$, i.e., to formulate the following optimization problem:

$$
\operatorname{minimize}\left(\frac{1}{\delta}\right) \text { subject to } \begin{cases}(a) & X^{-1} \geq \delta I \\ (b) & 19 .\end{cases}
$$

Remark 4. In the case of without disturbances i.e., $\bar{v}_{p}=0$ which yields $r=0$, the state trajectories of the system is exponential stable as stated in Definition 1.

\subsection{Discrete-time quasi-sliding mode controller}

In this subsection, a robust discrete-time quasi-sliding mode controller is proposed to force the sate trajectories to move monotonically towards the sliding surface designed in (12). After reaching the sliding surface, the state trajectories of the system cross the sliding surface for the first time and it will cross it again in every successive sampling period. This leads to a zigzag motion onto the sliding surface. This motion will be bounded inside a specified region the so-called quasi-sliding mode band (QSMB). Moreover, if the system trajectories reach the sliding surface, it will enter and stay within a QSMB [19].

Since the external disturbance $v(k)$ is assumed to be bounded so without loss of generality, we aslo assume that $\bar{C} \bar{D} v(k)$ will be bounded as follows

$$
d_{m} \leq \bar{C} \bar{D} v(k)=d(k) \leq d_{M},
$$

where $d(k)$ is a row vector and inequalities between real vectors will be understood componentwise.

Let us define the mean and spread of $d(k)$ as follows

$$
d_{0}=\frac{d_{m}+d_{M}}{2}, d_{1}=\frac{d_{M}-d_{m}}{2} .
$$

From sliding function 12 with design matrix $C=\left[\begin{array}{llll}C_{1} & C_{2} & \ldots C_{m}\end{array}\right]^{T}$ being obtained from (19), we have $s(k)=\left[\begin{array}{llll}s_{1}(k) & s_{2}(k) \ldots s_{m}(k)\end{array}\right]^{T}$, where $s_{i}(k)=-C_{i} z_{1}(k)+z_{2 i}(k)$ and $C_{i} \in$ $\mathbb{R}^{1 \times(n-m)}$ is a row vector.

Theorem 2. For given positive scalars $\bar{v}_{p}>0$ and $\alpha>1$, if there exist feasible solutions $X, K$ and $C$ for linear matrix inequality (LMI) (19), the state trajectories of underactuated mechanical system (10), (11) are driven towards the sliding surface designed in (12) in finite time with the following control law:

$$
u(k)=-(\bar{C} \bar{B})^{-1}\left[\bar{C} \bar{A} z(k)-\left(I-q T_{s}\right) s(k)+d_{0}+\left[\varepsilon T_{s}+d_{1}\right] \circ \operatorname{sgn}(s(k))\right],
$$

where $T_{s}$ is the sampling period, controller parameter $\varepsilon=\left[\varepsilon_{1}, \varepsilon_{2}, \ldots, \varepsilon_{m}\right]^{T}>0$ and $q=$ $\operatorname{diag}\left(q_{1}, q, \ldots, q_{m}\right)$ are chosen such that $0<q_{i} T_{s}<1, i=1,2, \ldots, m$.

Moreover, the quasi-sliding mode band is determined as

$$
\Delta_{i}=\frac{\varepsilon_{i} T_{s}}{1-q_{i} T_{s}} .
$$

Proof. To prove Theorem 2, we consider the reaching condition for the discrete-time quasi-sliding mode control proposed in [19], in which the sign of the incremental change of 
$\Delta s(k)=s(k+1)-s(k)$ should be opposite to the sign of $s(k)$.

From the designed sliding function $s(k)=\bar{C} z(k)$, we have

$$
\begin{aligned}
\Delta s(k) & =s(k+1)-s(k) \\
& =\bar{C} z(k+1)-\bar{C} z(k) \\
& =\bar{C}\left[(\bar{A}-I) z(k)+\bar{A}_{d} z(k-\tau(k))+\bar{B} u(k)+\bar{D} v(k)\right] .
\end{aligned}
$$

By substituting equation (30) into equation $(32)$, we obtain

$$
\Delta s(k)=-q T_{s} s(k)-\varepsilon T_{s} \circ \operatorname{sgn}(s(k))+\left[d(k)-d_{0}-d_{1} \circ \operatorname{sgn}(s(k))\right] .
$$

It is easy to see that

$$
\begin{aligned}
& d(k) \leq d_{0}+d_{1} \circ \operatorname{sgn}(s(k)), s(k)>0, \\
& d(k) \geq d_{0}+d_{1} \circ \operatorname{sgn}(s(k)), s(k)<0 .
\end{aligned}
$$

Thus, by judging the sign of the two terms constituting $\Delta s(k)$, we can see that the sign of the increment $\Delta s(k)$ of $(33)$ is always opposite to the sign of $s(k)$. Moreover, if $q_{i}>0$ is chosen such that $1-q_{i} T_{s}>0$, then the system trajectories reach the sliding surface $s(k)=0$ and remain within its QSMB, determined by [19] as,

$$
\Delta_{i}=\frac{\varepsilon_{i} T_{s}}{1-q_{i} T_{s}} .
$$

The proof is completed.

\section{PENDUBOT}

To illustrate the feasibility of the proposed approach, one of the popular underactuated mechanical system - the Pendubot is used. The Pendubot is basically a two-link planar robot manipulator with a DC motor that supplies a torque to the first link, while the second link is able to swing freely around its pivot. The Pendubot system is underactuated mechanical system since the angular acceleration of the second link cannot be controlled directly. It should be noted that the control problem of the Pendubot has similarities with that of the acrobot and the single and double inverted pendulums on a cart [27]. By neglecting friction and using the energy theorem, one can derive the following matrices:

$$
\begin{aligned}
M(q) & =\left[\begin{array}{cc}
\theta_{1}+\theta_{2}+2 \theta_{3} \cos \left(q_{2}\right) & \theta_{2}+\theta_{3} \cos \left(q_{2}\right) \\
\theta_{2}+\theta_{3} \cos \left(q_{2}\right) & \theta_{2}
\end{array}\right], \\
C(q, \dot{q}) & =\left[\begin{array}{cc}
-\theta_{3} \sin \left(q_{2}\right) \dot{q}_{2} & -\theta_{3} \sin \left(q_{2}\right)\left(\dot{q}_{2}+\dot{q}_{1}\right) \\
\theta_{3} \sin \left(q_{2}\right) \dot{q}_{1} & 0
\end{array}\right], \\
g(q) & =\left[\begin{array}{c}
\theta_{4} g \cos \left(q_{1}\right)+\theta_{5} g \cos \left(q_{1}+q_{2}\right) \\
\theta_{5} g \cos \left(q_{1}+q_{2}\right)
\end{array}\right], F=\left[\begin{array}{c}
u(t) \\
0
\end{array}\right],
\end{aligned}
$$

where

$$
\begin{aligned}
& \theta_{1}=m_{1} l_{c 1}^{2}+m_{2} l_{1}^{2}+J_{1}, \theta_{2}=m_{2} l_{c 2}^{2}+J_{2} \\
& \theta_{3}=m_{2} l_{1} l_{c 2}, \theta_{4}=m_{1} l_{c 1}+m_{2} l_{1}, \theta_{5}=m_{2} l_{c 2}
\end{aligned}
$$


in which $m_{1}, m_{2}, l_{1}, l_{2}$ are respectively the masses and the lengths of link 1 and link $2 ; l_{c 1}$ and $l_{c 2}$ are respectively the distances to the center mass of link 1 and link 2 from the suspension point; $J_{1}, J_{2}$ are the moments of inertia of link 1 and link 2 about their centroid; $q_{1}, q_{2}$ are the joint angle positions of link 1 and link $2 ; g$ is the gravitational acceleration; $v(t)$ is the external disturbances and $u(t)$ is the torque input to link 1 . The absence of the torque input to link 2 imposes a constraint on the system dynamics.

Now, we consider the following kinematic and dynamic parameters which have been considered in [27]

$$
\begin{array}{rlllll}
m_{1}=1.0367 & k g, l_{1}=0.1508 & m, m_{2}=0.5549 & k g, l_{2}=0.2667 & m, \\
d_{1}=0.1206 & m, J_{1}=0.0031 & k g . m^{2}, d_{2}=0.1135 \quad m, J_{2}=0.0035 \quad k g . m^{2} .
\end{array}
$$

The system parameters are identified as follows

$$
\begin{aligned}
& \theta_{1}=0.0308 \quad \mathrm{~kg} \cdot \mathrm{m}^{2}, \theta_{2}=0.0106 \quad \mathrm{~kg} \cdot \mathrm{m}^{2}, \theta_{3}=0.0095 \quad \mathrm{~kg} \cdot \mathrm{m}^{2} \\
& \theta_{4}=0.2087 \quad \mathrm{~kg} \cdot \mathrm{m}^{2}, \theta_{5}=0.0629 \quad \mathrm{~kg} \cdot \mathrm{m}^{2} .
\end{aligned}
$$

Now, we consider the external disturbance of the system as $v(k)=0.35 \sin (7 k)$. With $g=$ $9.81 \mathrm{~m} / \mathrm{s}^{2}$, by choosing the sampling period $T_{s}=0.01 \mathrm{sec}$, the parameter matrices of the system in equation (8) are obtained as:

$$
A=\left[\begin{array}{cccc}
1 & 0.01 & 0 & 0 \\
0.9895 & 1 & -0.0252 & 0 \\
0 & 0 & 1 & 0.01 \\
-0.6828 & 0 & 1.0472 & 1
\end{array}\right], B=\left[\begin{array}{c}
0 \\
-0.4479 \\
0 \\
0.8474
\end{array}\right], D=\left[\begin{array}{c}
0 \\
0.4479 \\
0 \\
-0.8474
\end{array}\right]
$$

and the transformation $T$ can be obtained from a singular value decomposition of matrix $B$ as:

$$
T=\left[\begin{array}{cccc}
-0.4673 & 0.7816 & 0 & 0.4131 \\
0 & 0 & 1 & 0 \\
0.8841 & 0.4131 & 0 & 0.2184 \\
0 & -0.4673 & 0 & 0.8841
\end{array}\right]
$$

With the above external disturbance, the controller parameters can be computed as

$$
d_{0}=0, d_{1}=-0.3355 .
$$

From Theorem 1 and Remark 2, by choosing $\alpha=1.005$, we obtain the sliding function as follows

$$
s(k)=\left[\begin{array}{llll}
23.5943 & -55.5143 & -69.1910 & 1
\end{array}\right] z(k) .
$$

The smallest possible radius of the ball $\mathcal{B}(0, r)$ is calculated as $r=0.35 \times 10^{-6}$. From Theorem 2, by choosing $q=4$ and $\varepsilon=10$, the robust discrete-time quasi-sliding mode controller is obtained of the form

$u(k)=-1.0433\left[\left[\begin{array}{llll}-25.2821 & 61.8113 & 74.3052 & 1.1534\end{array}\right]^{T} z(k)-0.96 s(k)-0.2355 \operatorname{sgn}(s(k))\right.$.

Consequently, the QSMB is determined as $\Delta=0.1042$. Simulation results for an initial condition of the system of $x(k)=\left[\begin{array}{llll}-\pi / 2 & 0 & \pi / 2 & 0\end{array}\right]$ (rad) are shown in Fig 1 and 2. It can 
be seen that the settling times for the angle position and angle velocity responses of link 1 and link 2 are $1.4 \mathrm{sec}$. However, due to the effects of external disturbances caused by friction and inertia moment, the angle velocities exhibits a small amplitude of oscillation between $\pm 0.5(\mathrm{rad} / \mathrm{s})$. Figure 2 depicts the plots of the switching function and input torque applied to the link 1 respectively.
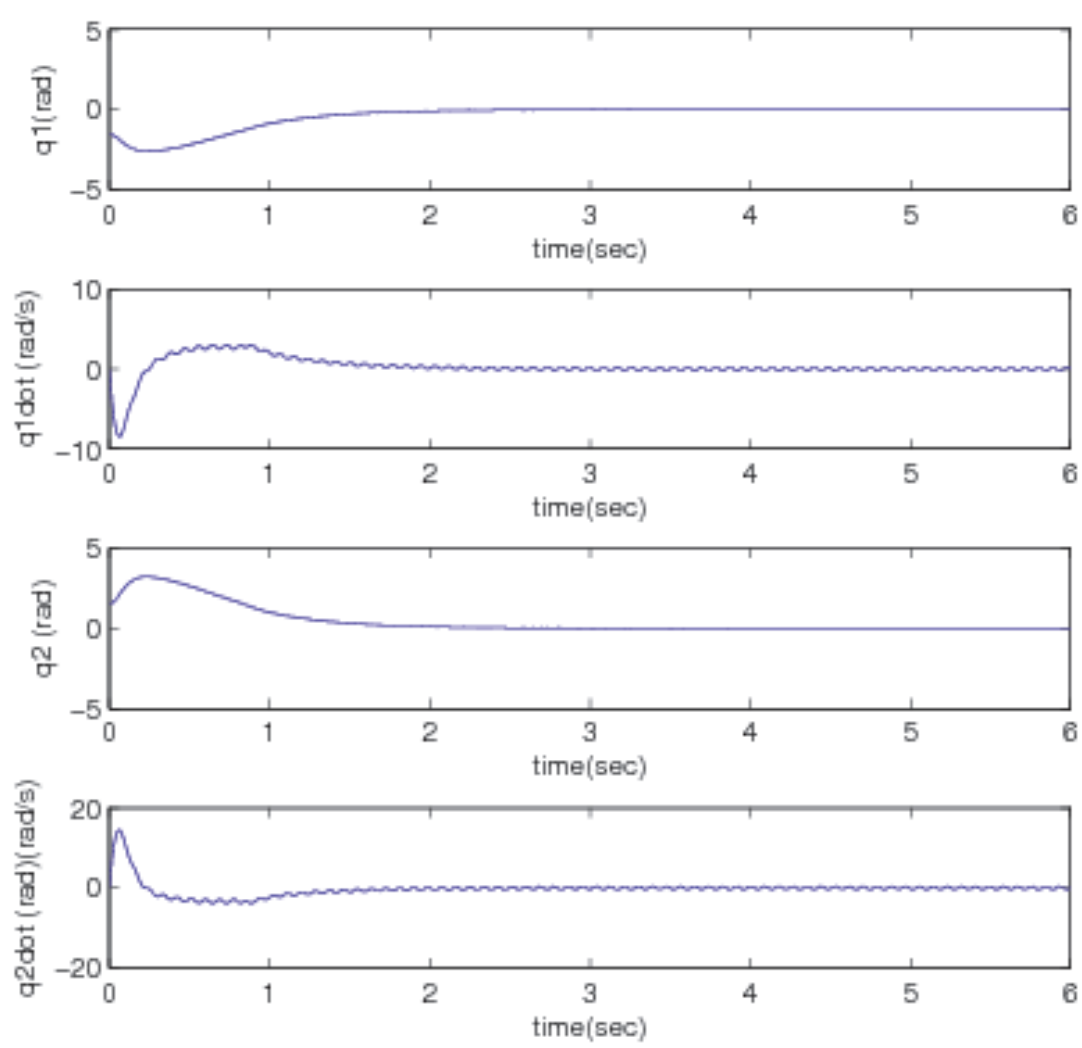

Fig. 1. Angle positions and velocities of joint 1 and 2

\section{CONCLUSION}

In this paper, the problem of robust discrete-time quasi-sliding mode control for a class of underactuated mechanical systems with bounded external disturbances has been addressed. Based on the Lyapunov method, a sufficient condition for the existence of a stable sliding surface has been derived in terms of a linear matrix inequality. This condition also guarantees that the effects of the external disturbances are suppressed when the system is in the sliding mode and the reduced-order sliding mode dynamics are exponentially convergent to a ball whose radius can be minimized. Finally, a robust discrete-time quasi-sliding mode controller is synthesized to drive system state trajectories towards the sliding surface in finite time and maintain it there afterwards. A case study of the Pendubot has been considered to demonstrate the feasibility of the proposed approach. 

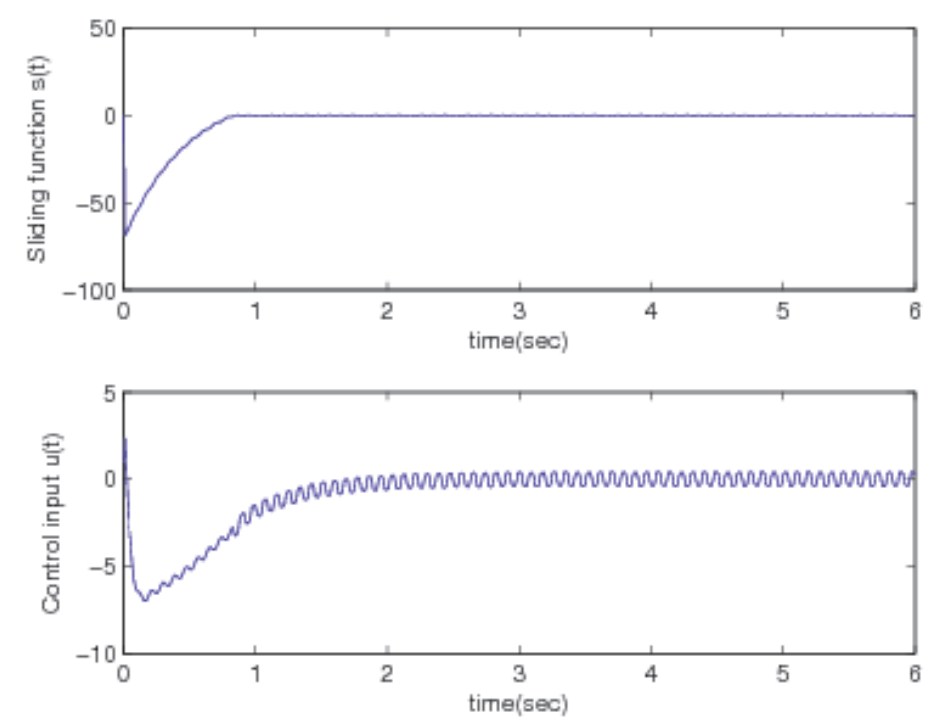

Fig. 2. Sliding function and control torque

\section{REFERENCES}

[1] Y. Liu, H. Yu, A survey of underactuated mechanical systems, IET Control Theory and Applications 7(7) (2013), 921-935.

[2] M.W. Spong, The swing-up control problem for the Acrobot, IEEE Control Systems Magazine 15(1) (1995), 49-55.

[3] M. Zhang and T.-J. Tarn, Hybrid control of the pendubot, IEEE/ASME Transactions on Mechatronics 7(1) (2002), 79-86.

[4] M. Zhang and T.-J. Tarn, A hybrid switching control strategy for nonlinear and underactuated mechanical systems, IEEE Transactions on Automatic Control 8(10) (Apr. 2003), 17771782 .

[5] I. Fantoni, R. Lozano, and M. W. Spong, Energy based control of the pendubot, IEEE Transactions on Automatic Control 45(4) (Apr. 2000), 725-729.

[6] O. Kolesnichenko and A. S. Shiriaev, Partial stabilization of underactuated Euler-Lagrange systems via a class of feedback transformations, System and Control Letters 45 (2002), 121-132.

[7] R. Ortega, M.W. Spong, F.G. Estern, G. Blankenstein, Stabilization of a class of underactuated mechanical systems via interconnection and damping assignment, IEEE Transactions on Automatic Control 47(8) (2002), 1128-1233.

[8] M.W. Spong, J.K. Holm, D. Lee, Passivity-based control of bipedal locomotion, IEEE Control Systems Magazine 14(2) (2007), 30-40.

[9] X. Xin, M. Kaneda, Analysis of the energy-based control for swinging up two pendulums, IEEE Transactions on Automatic Control 50(5) (2005), 679-684.

[10] X.Z. Lai, J.H. She, S.X. Yang, M. Wu, Comprehensive unified control strategy for underactuated two-link manipulators, IEEE Transactions on Systems, Man, and Cybernetics, Part B: Cybernetics 39(2) (2009), 389-398.

[11] K.D. Do, Z.P. Jiang, J. Pan, Underactuated ship global tracking under relaxed conditions, IEEE Transactions on Automatic Control 47(9) (2002), 1529-1536. 
[12] P. Batista, C. Silvestre, P. Oliveira, A sensor-based controller for homing of underactuated AUVs, IEEE Transactions on Robotics 25(3) (2009), 701-716.

[13] D. Chwa, Global tracking control of underactuated ships with input and velocity constraints using dynamic surface control method, IEEE Transactions on Control Systems Technology 19(9) (2011), 1357-1370.

[14] A.M. Bloch, N.E. Leonard, J.E. Marsden, Controlled Lagrangians and the stabilization of mechanical systems I: the first matching theorem, IEEE Transactions on Automatic Control 45(12) (2000), 2253-2270.

[15] A.M. Bloch, D.E. Chang, N.E. Leonard, J.E. Marsden, Controlled Lagrangians and the stabilization of mechanical systems II: potential shaping, IEEE Transactions on Automatic Control 46 (10) (2001) 1556-1571.

[16] V.I. Utkin, Variable structure control systems with sliding modes, IEEE Transactions on Automatic Control, 22(2) (1977), 212-222.

[17] H. Ashrafiuon, K.R. Muske, L.C. McNinch, R.A. Soltan, Sliding mode tracking control of surface vessels, IEEE Transactions on Industrial Eletronics, 55(11) (2008), 4004-4012.

[18] J. Huang, J.-H. Guan, T. Matsuno, T. Fukuda, K. Sekiyama, Sliding-mode velocity control of mobile-wheeled inverted-Pendulum systems, IEEE Transactions on Robotics, 26(4) (2010), 750-758.

[19] W. Gao, Y. Wang, A. Homaifa, Discrete-time variable structure control systems, IEEE Transactions on Industrial Electronics 42(2) (1995), 117-122.

[20] C.-C. Yih, Sliding mode control for swing-up and stabilization of the cart-pole underactuated system, Asian Journal of Control 15 (4) (2013), 1201-1214.

[21] R. Iriartea, L. T. Aguilara, L. Fridman, Second order sliding mode tracking controller for inertia wheel pendulum, Journal of the Franklin Institute 350(1) (2013), 92-106.

[22] E. Fridman and U. Shaked, On reachable sets for linear systems with delay and bounded peak inputs, Automatica 39(11) (2003), 2005-2010.

[23] S. Oucheriah, Robust exponential convergence of a class of linear delayed systems with bounded controllers and disturbances, Automatica 42(11) (2006), 1863-1867.

[24] N.D. That, P.T. Thanh, N.K. Quang and Q.P. Ha, Robust exponential stabilization of underactuated mechanical systems in the presence of bounded disturbances using sliding mode control, The International Conference on Control, Automation and Information Sciences, Nha Trang, Vietnam, 2013, 181-186.

[25] N.D. That, Robust discrete-time quasi-sliding mode control design of the Pedubot in the presence of bounded disturbances, The 2nd Vietnam Conference on Control and Automation, Da Nang, Vietnam, 2013.

[26] N.D. That, P.T. Nam and Q.P. Ha, Reachable set bounding for linear discrete-time systems with delays and bounded disturbances, Journal of Optimization Theory and Applications 157 (1) (2013), 96-107.

[27] T. Albahkali, R. Mukherjee, and T. Das, Swing-up control of the Pendubot: an impulsemomentum approach, IEEE Transactions on Robotics 25 (4) (2009), 975-982.

Received on February 26, 2013

Revised on October 16, 2013 